\title{
Parametric hysteresis in power amplifiers
}

\author{
Jesús de Cos, Almudena Suárez, and José Angel García \\ Department of Communications Engineering. University of Cantabria, Santander, 39012, Spain
}

\begin{abstract}
Parametric hysteresis in power amplifiers is investigated, studying the causes of this phenomenon and providing an efficient methodology for its prediction and elimination. As will be demonstrated, in MESFET and HEMT devices it is caused by a nonlinear resonance of the device input capacitance under near optimum input matching conditions. Bifurcation loci are used to evaluate the impact of the phenomenon under variation of critical design parameters. All the tests have been carried out in a Class-E GaN PA with measured $86.8 \%$ PAE and $12.4 \mathrm{~W}$ output power at $0.9 \mathrm{GHz}$.

Index Terms - Bifurcation, class-E, GaN, harmonic balance (HB), hysteresis, PAE, power amplifier, stability.
\end{abstract}

\section{INTRODUCTION}

Hysteresis or jumps in the power transfer curve of power amplifiers (PA) are an undesired phenomenon that has been reported in [1], where a detailed analysis of a Class-E PA was presented. From a geometrical viewpoint, the hysteresis is due to the presence of turning points or infinite slope points in the solution curves, which could be detected in [1-2] by means of an auxiliary generator (AG) introduced into the commercial harmonic balance (HB) software. In [2] hysteresis could be related to the gate current from impact ionization coupling with the bias resistor. However, when the hysteresis is observed only in a large-signal regime, a different mechanism should be involved, which has not been investigated in any previous work to our knowledge. Here, the hysteresis will be related to the nonlinear resonance of one of the device capacitance(s) under near optimum matching conditions. As will be shown, the subnetwork containing the nonlinear capacitance can be approximately described with the Mathieu equation [3-4], having two major resonances: one at the subharmonic frequency and one at the input frequency, the latter being responsible for the infinite slope points. After this investigation, an efficient simulation method will be presented. In [1] parameter switching and optimization had to be applied to obtain the multivalued solution curves. The turning point loci were calculated through detection of the singular points of the Jacobian matrix of the AG admittance function. This required an involved estimation of the determinant threshold to ensure sufficient analysis accuracy. The new method is developed from the recently published procedure [5] for studying injection-locked oscillators. It avoids all of these requirements and is based on the calculation of an outer-tier admittance function in commercial HB. The investigation of the hysteresis phenomena and the methodologies for its analysis and suppression will be illustrated through application to a Class-E GaN PA at $0.9 \mathrm{GHz}$ with a measured close to the state-of-the-art poweradded efficiency of $86.8 \%$ and $12.4 \mathrm{~W}$ of output power.

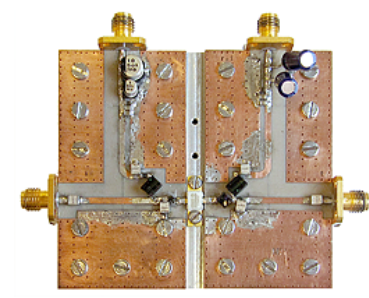

a)

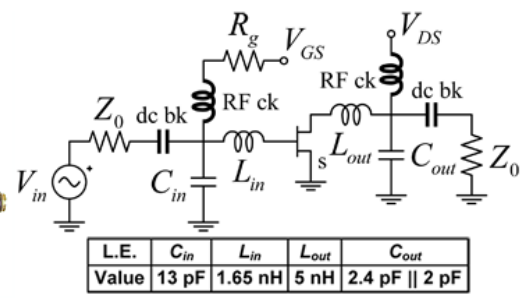

Fig. 1. The demonstrator is a highly efficient Class-E power amplifier at $0.9 \mathrm{GHz}$. (a) Photograph. (b) Schematic (values in the table are for Coilcraft Air Core inductors and ATC 100B capacitors).

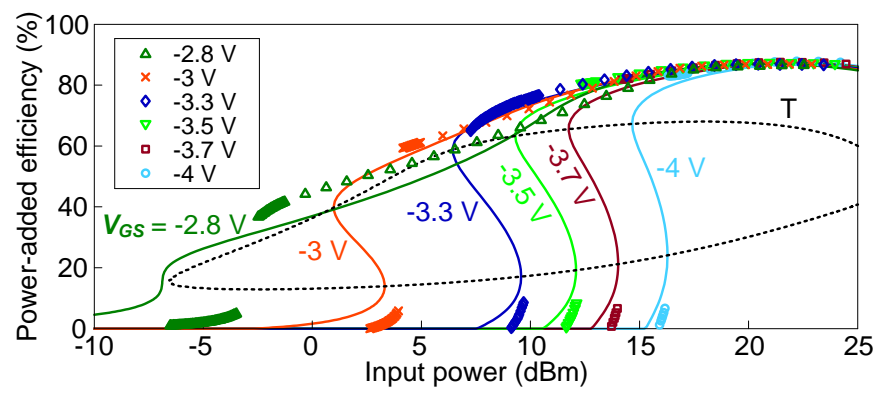

Fig. 2. PAE versus $P_{a v}$ for different $V_{G S}$ values. The turning point locus $T$ has been included (dashed line). Solid lines are simulated results and symbols are measurements.

\section{PARAMETRIC HYSTERESIS}

The Class-E PA in Fig. 1, based on Cree's GaN HEMT CGH35030F, takes advantage of $L_{\text {out }}$ parasitics to force high reactance terminations at the harmonics [6], while the optimum drain impedance at the fundamental is adjusted through $C_{\text {out }}$, following the solution proposed in [7]. The typical single low-pass section used to match the input improves the PA gain in such a way that the power-added efficiency (PAE) gets very close to the drain efficiency value.

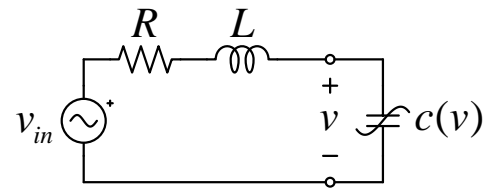

Fig. 3. A simple network is considered to match the input of the power amplifier, resulting in an R-L-diode circuit. 
The PAE curves are represented in Fig. 2 for various gate bias voltages $\left(V_{G S}\right)$. The two turning points in each curve give rise to hysteresis when increasing and decreasing the input power $\left(P_{a v}\right)$. The phenomenon still occurs when shortcircuiting the drain and source terminals, so it can only be due to the nonlinear gate-to-source capacitance. Indeed, the input impedance of MESFET and HEMT devices has usually a small real part and a negative reactance dominated by the gate-to-source capacitance [8]. Therefore, the simplest matching network is a resistance and an inductance connected in series, which gives rise to the R-L-diode circuit in Fig. 3. Clearly, the actual input matching network will be different, but some important conclusions can be derived from this simple circuit. In terms of the diode charge $q$, the circuit is ruled by the following nonlinear differential equation:

$$
v_{i n}(t)=L \ddot{q}(t)+R \dot{q}(t)+v(q) .
$$

Considering for simplicity a first-order Taylor expansion of $c(v)$ and sinusoidal voltage at the input frequency $\omega_{\text {in }}$ :

$$
c(t)=C_{0}-\Delta C \cos \left(\omega_{i n} t\right),
$$

where $\Delta C / C_{0}<<1$ is assumed. To focus the analysis on the resonator, the input source will be eliminated $\left(v_{i n}=0\right)$ as well as the damping term resulting from the resistance $R$, which leads to the undamped linear Mathieu equation [4]:

$$
\left\{\begin{array}{l}
\dot{q}=\imath \\
i=-\omega_{0}^{2}\left[1+\beta \cos \left(\omega_{i n} t\right)\right] q
\end{array},\right.
$$

where $t(t)$ is the loop current, $\omega_{0}{ }^{2}=1 /\left(L C_{0}\right), \beta=\Delta C / C_{0}$ and the approximation $1 /(1-\varepsilon) \approx 1+\varepsilon$ when $\varepsilon<<0$ has been used. The general solution of (3) can be expressed as a combination of two linearly independent solutions:

$$
\left[\begin{array}{l}
q(t) \\
l(t)
\end{array}\right]=c_{1}\left[\begin{array}{l}
q_{1}(t) \\
l_{1}(t)
\end{array}\right]+c_{2}\left[\begin{array}{l}
q_{2}(t) \\
l_{2}(t)
\end{array}\right]=\underbrace{\left[\begin{array}{ll}
q_{1}(t) & q_{2}(t) \\
l_{1}(t) & l_{2}(t)
\end{array}\right]}_{X(t)}\left[\begin{array}{l}
c_{1} \\
c_{2}
\end{array}\right] .
$$

If the pair of independent solutions are chosen such that $X(0)=I$, the eigenvalues of $X(T)$, where $T$ is the period of the steady state solution, are the characteristic Floquet multipliers [3-4], which determine the stability of the periodic solution. It is easily seen that the time derivative of the determinant $W(t)=\operatorname{det}[X(t)]$ is zero and thus $W$ is time invariant. At $t=0$, one has $W(0)=1$, since $X(0)=I$. Therefore, the two multipliers fulfill $\operatorname{det}[X(T)]=m_{1} m_{2}=1$. For stability, all the multipliers must have magnitude smaller than one. Thus, instability would be obtained for two different and real multipliers of the same sign. For two multipliers equal to +1 a singularity in the solution curve, associated with a turning point, would be obtained. The case $m_{1,2}=-1$ (frequency division by 2) is well known [3-4,8-9]. However, the case $m_{1,2}=+1$ seems to be overlooked, although it is the cause of the hysteresis. To see this one must take into account the relationship between multipliers and poles $(p)$, which has the form $m=\exp (p T)$. Therefore, a multiplier $m=1$ implies a pole $p$ at zero, leading to a singularity in the solution curve. Equation (3) is unrealistic since the damping term $\gamma$ has been neglected. In the presence of $\gamma$, instability is only possible with a nonlinear capacitance. For this nonlinear analysis $q(v)$ will be replaced with its describing function, assuming an input voltage waveform $v_{i n}(\mathrm{t})=V_{\text {in }} \cos \left(\omega_{\text {in }} t+\phi\right)$, which leads to the following steady-state equation in the frequency domain:

$$
V-V_{i n} e^{j \phi}+j \omega_{i n} R Q(V)-\omega_{i n}^{2} L Q(V)=0 .
$$

The Jacobian matrix of the complex equation (5) is calculated with respect to $V$ and $\phi$. The singularity condition is:

$$
\begin{aligned}
& \left|\begin{array}{cc}
1-\omega_{i n}^{2} L Q^{\prime} & V_{i n} \sin \phi \\
\omega_{i n} R Q^{\prime} & -V_{i n} \cos \phi
\end{array}\right|= \\
& =-\left(\omega_{i n}^{2} L Q^{\prime}-1\right)\left(\omega_{i n}^{2} L Q-V\right)-\left(\omega_{i n} R\right)^{2} Q^{\prime} Q=0
\end{aligned},
$$

where $Q^{\prime}$ is the derivative of $Q(V)$. Condition (6) can only be fulfilled for a nonlinear characteristic $Q(V)$, since a linear capacitance leads to $V=0$. The singularity is more likely to occur under good input matching conditions, since, as derived from (6), it requires the nonlinear resonance of the device capacitance with the inductor. The large-signal reflection coefficient is obtained from a generalization of the smallsignal $S_{11}$ definition: $\Gamma=\left(V_{\text {in }}-Z_{0}^{*} I_{\text {in }}\right) /\left(V_{\text {in }}+Z_{0} I_{\text {in }}\right)$. The magnitude of $\Gamma$ has been calculated with (5) considering $V_{G S}=-4.6 \mathrm{~V}$. In Fig. 4 it has been represented versus $P_{a v}$ in dashdot line where it can be compared with results obtained with the simulation method of Section III and with measurements of the PA in Fig. 1 when short-circuiting the drain and source terminals.

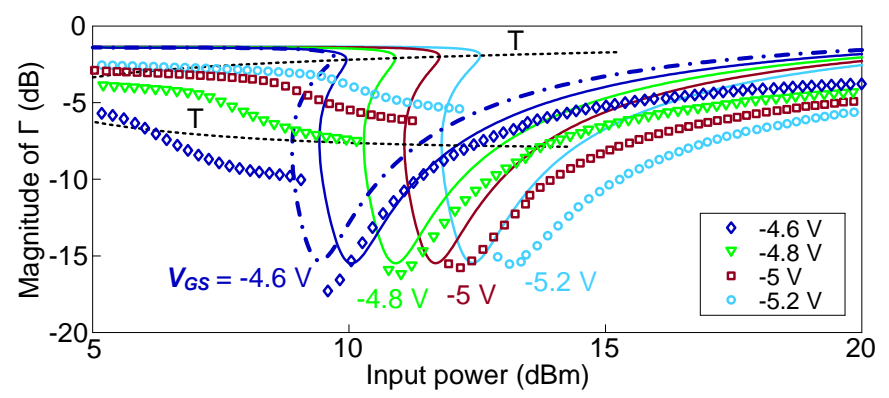

Fig. 4. Magnitude of $\Gamma$ versus $P_{a v}$ for different $V_{G S}$ values. The dashdot curve is calculated with (5). The turning point locus $T$ has been included (dashed line). Solid lines are simulated results and symbols are measurements.

\section{OUTER-TIER METHOD}

The nonlinear simulation method is based on the admittance description of the PA at the gate terminal, as depicted in Fig. 5(a). The Norton equivalent of the input network [Fig. 5(b)] is calculated from its scattering parameters, together with the admittance function $Y_{g s}$ seen from the gate terminal. This last function is obtained from a two-tier HB resolution of the circuit obtained by suppressing the input 
current source and connecting an AG between the gate and source terminals:

$$
Y_{g s}=I_{A G, 1} / V_{A G},
$$

where $I_{A G, 1}$ is the first harmonic of the AG current and $V_{A G}$ is the AG voltage. Equation (7) is solved with the pure HB system, and with as many harmonic components as necessary, as an inner tier. The high-pass filter included in Fig. 5(c) allows for proper termination of higher harmonics.

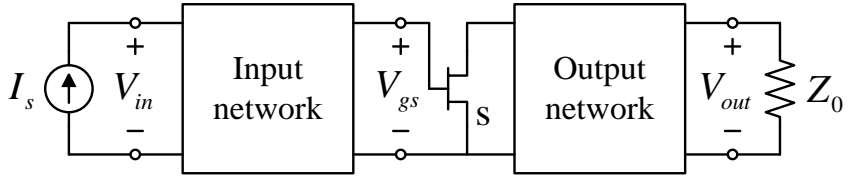

a)

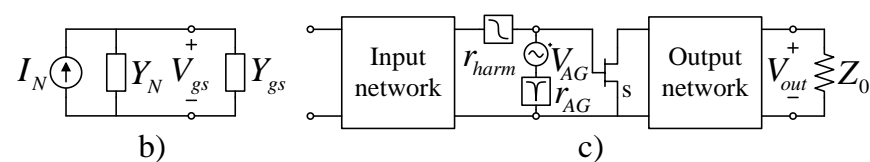

Fig. 5. (a) Schematic of a power amplifier at the gate terminal. The impedance $Z_{0}$ of the Norton representation of the input generator is included inside the input network. (b) Norton equivalent at the gate terminal. (c) Circuit for obtaining the gate admittance function $Y$ gs.

Applying Kirchhoff's current law to the circuit in Fig. 5(b) and substituting with the expressions of the Norton equivalent current $I_{N}$ and admittance $Y_{N}$, one obtains the equation:

$$
I_{s} e^{j \phi_{g s}}=Y_{e q} V_{g s},
$$

where the equivalent admittance is

$$
Y_{e q}=\frac{Y_{0}\left[1-S_{11}+\left(\Delta-S_{22}\right)\right]+Y_{g s}\left[1-S_{11}-\left(\Delta-S_{22}\right)\right]}{2 S_{21}},
$$

$\Delta=S_{11} S_{22}-S_{12} S_{21}, Y_{0}=1 / Z_{0}$ is the characteristic admittance, $V_{g s}$ is the gate voltage amplitude and $\phi_{g s}$ is the phase shift between $I_{N}$ and the gate voltage. The input current $I_{s}$ is finally obtained from the real and imaginary parts of (8):

$$
I_{s}=\left|Y_{e q}\right| V_{g s} .
$$

The available input power is $P_{a v}=Z_{0} I_{s}^{2} / 8$. The input voltage is calculated from the Norton equivalent:

$$
V_{\text {in }}=\frac{Z_{0}\left[1+S_{11}+\left(\Delta+S_{22}\right)\right]+2 S_{12} / Y_{e q}}{1-S_{11}-\left(\Delta-S_{22}\right)} I_{s} .
$$

The current provided by the input generator is $I_{i n}=I_{s}-V_{i n} / Z_{0}$.

To trace the solution curves versus a parameter $\mu$ different from $P_{a v}$ one must combine the results of two independent simulations: S-parameters of the input network and an $\mathrm{HB}$ double sweep in $\mu$ and $V_{A G}$ of the circuit in Fig. 5(c). The above method has been applied to obtain the multivalued curves of Figs. 2, 4, with $N H=15$ harmonic terms.
Measurements are superimposed matching the simulated results. Discrepancies come from modeling inaccuracies.

The scalar current equation (10) describes a surface on the plane $\mu, V_{g s}$. Let the function

$$
\Sigma\left(\mu, V_{g s}\right)=\left|Y_{e q}\right| V_{g s}
$$

be considered. The turning point locus is the set of points of the solution curves satisfying infinite slope and are given by:

$$
T=\left\{\left(\mu, V_{g s}\right) \in \mathbb{R}^{2}: \partial \Sigma\left(\mu, V_{g s}\right) / \partial V_{g s}=0\right\},
$$

where the derivative $\partial \Sigma / \partial V_{g s}$ is calculated numerically from (12). The locus (13) is computed attaching $P_{a v}$ as independent variable of $\partial \Sigma / \partial V_{g s}$ and calculating the zero-level contour. This method has been applied to obtain the loci superimposed in dashed line in Figs. 2, 4, which pass through all the turning points of the solution curves. It has also been used to evaluate the effect of the input capacitor $C_{i n}$ on the hysteresis phenomenon, as shown in Fig. 6, where the loci are obtained in the plane $V_{G S}, V_{g s}$. Using (13) multiple loci are obtained in a straightforward manner in Fig. 6 . With $C_{i n}=11 \mathrm{pF}$ the curves do not exhibit hysteresis, as shown in Fig. 7 where measurements are also superimposed.

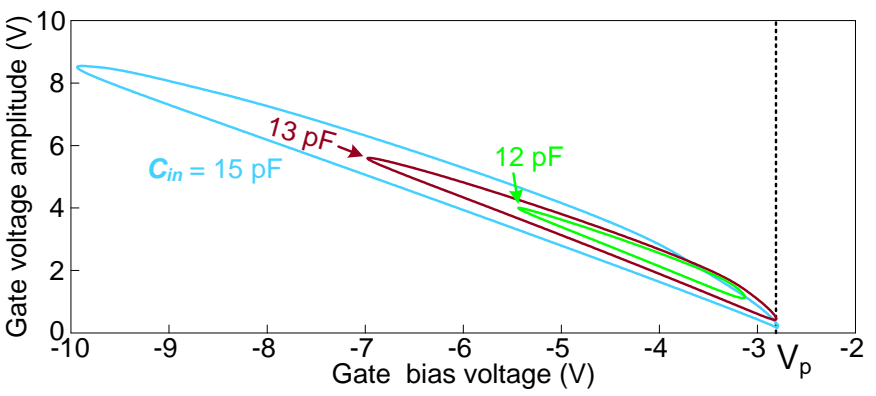

Fig. 6. Turning point loci in the plane $V_{G S}, V_{g s}$ for different $C_{i n}$ values. For $C_{i n}=11 \mathrm{pF}$ no locus is obtained.

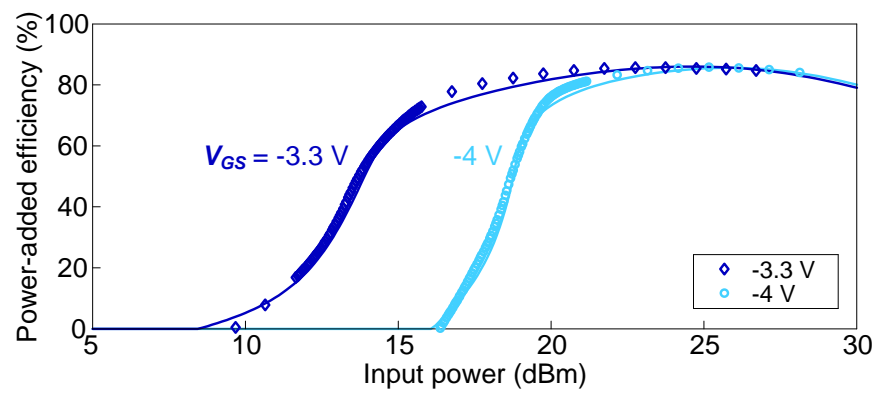

Fig. 7. PAE versus $P_{a v}$ for different $V_{G S}$ values. The hysteresis has been eliminated by lowering $C_{i n}$. Solid lines are simulated results and symbols are measurements.

\section{CONCLUSION}

An in-depth investigation of hysteresis in power amplifiers has been presented, demonstrating that the input network dynamics can be approximately modeled with the Mathieu 
equation. A novel method to obtain the multivalued solution curves and turning point loci has also been shown. The analysis has been applied to a Class-E GaN PA at $0.9 \mathrm{GHz}$ with a measured close to the state-of-the-art power-added efficiency of $86.8 \%$ and $12.4 \mathrm{~W}$ of output power.

\section{ACKNOWLEDGEMENT}

This work was supported by the Spanish Ministry of Economy and Competitiveness under Contract TEC201129264-C03-01 and FEDER co-funded Contract TEC201129126-C03-01, and by the Predoctoral Fellowship for Researchers in Training of the University of Cantabria and the

Regional Ministry of Education of the Government of Cantabria. The authors would like to thank M.N. Ruiz, University of Cantabria, for her assistance with measurements.

\section{REFERENCES}

[1] S. Jeon, A. Suárez, and D.B. Rutledge, "Analysis and elimination of hysteresis and noisy precursors in power amplifiers," IEEE Trans. Microw. Theory Techn., vol. 54, no. 3, pp. 1096-1106, Mar. 2006.

[2] N.-Ch.Kuo, P.-S.Chi,A. Suárez, J.-L.Kuo, P.-Ch. Huang, Z.M.Tsai, and H. Wang, "DC/RF hysteresis in microwave pHEMT amplifier induced by gate current-diagnosis and elimination,” IEEE Trans. Microw. Theory Techn., vol. 59, no. 11, pp. 2919-2930, Nov. 2011.

[3] A. Suárez, Analysis and Design of Autonomous Microwave Circuits. Hoboken, New Jersey: John Wiley \& Sons, 2009.

[4] J. Guckenheimer and P. Holmes, Nonlinear Oscillations, Dynamic Systems, and Bifurcations of Vector Fields. New York: Springer-Verlag, 1983.

[5] J. de Cos and A. Suárez, "Efficient simulation of solution curves and bifurcation loci in injection-locked oscillators," IEEE Trans. Microw. Theory Techn., vol. 63, no. 1, pp. 181-197, Jan. 2015.

[6] J.A. García, R. Marante, and M.N. Ruiz, "GaN HEMT Class E resonant topologies for UHF DC/DC power conversion," IEEE Trans. Microw. Theory Techn., vol. 60, no. 12, pp. 4220-4229, Dec. 2012.

[7] R. Negra and W. Bächtold, "Lumped-element load-network design for class-E power amplifiers," IEEE Trans. Microw. Theory Techn., vol. 54, no. 6, pp. 2684-2690, Jun. 2006.

[8] A. Grebennikov, N. O. Sokal, and M. J. Franco, Switchmode RF and Microwave Power Amplifiers, 2nd Edition. Waltham, Massachusetts: Academic Press, 2012.

[9] M. and J. Golio, RF and Microwave Circuits, Measurements, and Modeling. Boca Raton, Florida: CRC Press, 2007. 